\title{
Feasibility of Quantum Computers in Cryogenic Systems
}

\author{
${ }^{1}$ Viplove Divyasheesh, ${ }^{2}$ Rakesh Jain \\ ${ }^{1,2}$ Assistant Professor,IIST Indore
}

\begin{abstract}
Quantum computers consist of a quantum processor - sets of quantum bits or qubits operating at an extremely low temperature - and a classical electronic controller to read out and control the processor. The machines utilize the unusual properties of matter at extremely small scales - the fact that a qubit, can represent " 1 " and " 0 " at the same time, a phenomenon known as superposition. (In traditional digital computing, transistors in silicon chips can exist in one of two states represented in binary by a 1 or 0 not both).

Under the right conditions, computations carried out with qubits are equivalent to numerous classical computations performed in parallel, thus greatly enhancing computing power compared to today's powerful supercomputers and the ability to solve complex problems without the sort of experiments necessary to generate quantum phenomena.

the technology is unstable and needs to be stored in a cool environment for faster and more secure operation.In this paper, we discuss the possibility of integrating quantum computers with electronics and deep cryogenic temperatures.
\end{abstract}

\section{Index Terms-Quantum Computers, qubits, superposition, cryogenic.}

\section{Introduction}

A quantum computer is any device for computation that makes direct use of distinctively quantum mechanical phenomena, such as superposition and entanglement, to perform operations on data. In a classical (or conventional) computer, information is stored as bits; in a quantum computer, it is stored as qubits (quantum bits).

The basic principle of quantum computation is that the quantum properties can be used to represent and structure data, and that quantum mechanisms can be devised and built to perform operations with this data. Although quantum computing is still in its infancy, experiments have been carried out in which quantum computational operations were executed on a very small number of qubits.

Quantum computers are very unstable and thus are not being used in wide domains yet.They require them to be operated on very low temperatures. And this is what the paper tires to explore : feasibility of quantum computing at cryogenic level.

\section{Cryogenics}

Cryogenics is a branch of physics that deals with the effects of very low temperatures on a given system. Many things like LHC(Large Hadron Collider), superconductivity and supermagnetism could not have happened without cryogenics.

Cryogenic computer and cryogenic processor architecture are all intersections of domains of computers and cryogenics.

One of the requirements for a scalable quantum computer is superconducting circuit design. And that alone is not possible without the help of cryogenics.Also to create an Ion trap one must utilize the principles of cryogenic systems as well as computing.

\section{III. challenges for the establishment of a quantum computing environment}

The following things are to be kept in mind while creating a cryogenic quantum computer :- 
- Even the slightest of mechanical vibrations can destroy a quantum computing environment.

- Design of an Ion trap requires high vacuum conditions.

- Cryogenic conditions are required to absorb thermal vibrations as well.

- Power fluctuations and even the Zeeman effect can also disturb the quantum system.

\section{Factors responsible for a feasible quantum computing environment}

Various factors and their importance lies in the establishment of a ultra-stable quantum computing environment. There can be local differences, but at the quantum level, everything counts that makes even the slightest impact. Thus it is important to keep the following factors in mind while designing a Quantum computing environment.

\begin{tabular}{|l|l|}
\hline \multicolumn{1}{|c|}{ FACTOR } & \multicolumn{1}{|c|}{ ROLE } \\
\hline Low Vibration & $\begin{array}{l}\text { Preserves states of } \\
\text { qu-bits }\end{array}$ \\
\hline $\begin{array}{l}\text { Low } \\
\text { temperatures }(<-269.15 \\
\text { C) }\end{array}$ & Quick sampling \\
\hline Low working distance & $\begin{array}{l}\text { Minimal experimental } \\
\text { setup and maximal data } \\
\text { collection time; quick } \\
\text { and easy integration. }\end{array}$ \\
\hline Optical Access & Large sample space \\
\hline Electrical access & $\begin{array}{l}\text { Low loss, higher } \\
\text { frequency signal }\end{array}$ \\
\hline High Vacuum & $\begin{array}{l}\text { Stable environment for } \\
\text { study of trapped ions }\end{array}$ \\
\hline Magnetic Shielding & $\begin{array}{l}\text { Reduce the influence of } \\
\text { stray electromagnetic } \\
\text { radiation }\end{array}$ \\
\hline
\end{tabular}

\section{Conclusion}

Quantum computers are a way of the future. There are still 15-20 years left in implementing their commercial variants. But the technology requires cryogenic systems to begin with and thus cryo-environment are a viability for quantum computers to exist and to be studied. Plus, right now the only thing we can do is to study what lies in front and wait for what comes
ahead.But one thing is for sure, cryogenics will have a major role to play.

\section{References}

[1] Johnson, K. G. et al. Active Stabilization of Ion Trap Radiofrequency Potentials. Review of Scientific Instruments 87, 53110 (2016).

[2] Brown, K. R., Kim, J. \& Monroe, C. Co-Designing a Scalable Quantum Computer with Trapped Atomic Ions. arXiv:1602.02840 [quant-ph] (2016).

[3] Debnath, S. et al. Demonstration of a small programmable quantum computer with atomic qubits. Nature 536, 63-66 (2016).

[4] Steane, A. M. The Ion Trap Quantum Information Processor. Applied Physics B: Lasers and Optics 64, 623-643 (1997).

[5] https://www.montanainstruments.com/Applicatio ns/Quantum-Computing-in-Cryogenic-Systems

[6] https://www.pioneeringminds.com/cryogenics-an d-quantum-computing/ 